\title{
Personalizing University Email toward User Acceptance: An Initial Study
}

\author{
Bshaer Alwagadani \\ Department of Information Systems \\ King Abdulaziz University \\ Jeddah, Kingdom of Saudi Arabia
}

\author{
Khalid Alomar \\ Department of Information Systems \\ King Abdulaziz University, \\ Jeddah, Kingdom of Saudi Arabia
}

\begin{abstract}
The purpose of this paper is to examine students and faculty members commitment of using university email in Saudi Arabia, using online survey at King Abdulaziz University. The paper describes an initial study conducted before the development of an experimental personalized email platform to investigate these issues. The survey targets 1847 students (1359 female and 488 male), and 101 faculty members (58 female, and 43 male). The results show that only $23 \%$ of students are using the university email to communicate regularly, unlike the faculty members who shows $80 \%$ commitment. Furthermore, the evidence from this study suggests that applying personalized techniques has a strong and positive impact on both types of respondents, and considered as a clue for a potential system improvement to increase users' commitment of the university email.
\end{abstract}

\section{Keywords}

University email; personalization; adaptive system; informal email; acceptance

\section{INTRODUCTION}

Today, effective communication between students and faculty members as a means of improving learning processes is one of the vital domains in the field of educational systems [1]. Email technology is described as an active interaction tool of Computer-Mediated-Communication (CMC) [2]. The widespread usage of email technology in Middle Eastern countries such as Saudi Arabia led to facilitate everyday work in organizations, companies, and universities [3]. This sort of online communications has extremely used with other technologies types in many higher educational sectors [2].

Latest studies have highlighted the importance of communication and interaction between students and faculty members in raising the academic performance [4]. Students tend to use the email as an essential channel to communicate with their faculty members [5]. Furthermore, they prefer to contact their professors and work on their business in a formal way through their university email account. On the other hand, they still using other informal mail for social communications [6].

Despite prior facts, recently university email accounts are replaced by social networking sites in a high percentage of institutional students [7]. The tendency of students to use social networks and informal e-mail accounts in communication for an academic purpose is more than that of faculty members, who in turn prefer traditional communication such as university e-mail [8, 9]. Moreover, students may not check their university email frequently, and this will cause missing some important information or announcements from the college [6].
In general, it's hard to obtain accurate data about student committed to use university email. It appears from the investigations as mentioned above that numerous study has been conducted to assess the effects of formal communication absence. However, many universities still force the students to use their closed institutional communications such as email. So that, various educational institutions emphasize the importance of developing new models of learnings technologies to meet students' needs [10]. It is claimed that if the administrators apply useful personalized technology through education and communication systems, students will be more engaged with online learning environment [2].

This study aims to experimentally investigate the reasons for low utilization rate of university email, and illustrate system defects that must be considered when developing adaptive email framework. In addition to investigating personalized system ability to enhance university email usage by students and faculty members. It is suggested to integrate both adaptive and adaptable technique to design email system to be more personalized and convenient to the user demand [11]

\section{PROBLEM STATEMENT AND RESEARCH QUESTIONS}

Modern communicating technologies play a significant role in raising the efficiency of academic performance among students and faculty members [4]. Recent studies have focused on the development of this aspect of many universities and educational institutions [2]. University's email is one of these tools, and it is considered as the primary channel that has been approved by the University to communicate and send necessary information and contents between student and faculty members [10]. Therefore, it has been observed that most of the students would not prefer to use it to communicate and place it as a primary reference for any purpose $[7,12]$. In summary, there is a strong need to identify the reasons and factors that affect the commitment of using King Abdulaziz University email system, and whether adaptive or adaptable techniques will improve the usage of university email system and raise its efficiency and increase better communication through it. 


\subsection{Research question}

The present study focus on whether the personalized email system will improve students' commitment to using university email. Accordingly, the following questions have to be addressed to answer the main previous question:

1. What are the reasons that affected King Abdulaziz University users to avoid using formal university email?

2. What are the useful personalized and customized techniques to develop the best-personalized prototype system for university email?

3. In what ways does new personalized email system have a positive effect on rising student acceptance to use university email in KAU?

\subsection{Objectives}

The study will be valuable:

1. To determine the current system defects from the perspective of students and faculty members and define their preference for a new proposed system.

2. To identify which personalized and customized tool to develop a new adaptive system

3. To develop a new prototype for university email system depending on previous factors.

4. To increase effective communication between students and faculty members in KAU through adopting an efficient, personalized system.

\section{LITERATURE REVIEW}

Predicting user behavior have taken great importance in many popular kinds of research to improve personalization in email systems. Singh and Chetty [13], presented RANSAC; a new design architecture of system personalization for electronic email. The architecture is constructed by using Gradient Boost Trees model in addition to Optimized Pruned Extreme Learning Machines. It is implemented in the user database from OURDAL. The accurate results from conducted deep data analysis through the proposed architecture, is helpful to predict users behavior that led them to increase clicking on some e-mail messages.

Most existing email systems give great importance to arrange several incoming email message technique, which saves many users from time-consuming in recognizing their messages. Srivastava and Goyal proposed an email response for university system which synchronizes different mailboxes by data synchronization that can transfer to a single platform. In addition, the system personalizes emails depending on the user's requirement by arranging the respective emails according to their feedback through using natural language processing and content extraction. The software proposed have not only personalized and synchronized technique but also create an automatic constant reply [14]. Karthik and Ponnusamy, attempted to identify student and parent emails for educational institutions system by expressing their emotions through a unique method. It classifies incoming emails into three groups (positive, negative, others). The system uses an emotional dictionary that contains descriptive phrases and words as a central component, which is classified by SVD and KNN algorithm. The system is adaptive and could adapt new several words that describe the human emotions [15]. Sethi et al. presented a personalized intelligent email system, which arranges emails depending on users' preferences. The application serves people who deal with a large number of emails by enabling them to choose personal folders labels in the mailbox. Naïve Bayes classification was the best to classify incoming mails to each folder among other classifiers. This approach was helping to shift the focus from customization to personalization concept which concentrates on providing the control to the user [16].

Some useful systems have helped the user to retrieve some certain messages or find an exact receiver. Serim et al. [17] presented mailVis visual interface system which enables the user to retrieve and re-find previous emails in an efficient way. Re-finding could be described as a difficult and timeconsuming process, because it requires searching, scanning, and modifying multiple emails to find the desired message. mailVis derives its advantage from having several options and memory clues to facilitate a searching process for the user. The designer adds filter swipe, an interactive technique which integrates selection and filtering tasks into one single action in a dataset [17]. Regarding finding the exact receiver, there is basic functionality to adapt email messaging systems for this purpose. Brzezinski and Dian, proposed one type of this technology. MEME system clustering is based on users educational background. This function exceeds the capability of just sending messages via email. It evaluates information and generates knowledge based on system consumer profile and his preferences. Its functionality enables the user to find the exact receiver and delivers targeted email messages with a high efficiency [18].

Spam filtering is another important basic technique that must be considered when designing email system to detect unsolicited email messages. Mao et al. proposed an adaptive email system for spam filtering based on identifying the intentions of email senders by E-mail Word Social Network (EWSN) mechanism and artificial immune system. The EWSN was created based on isolated interested words and information gathered from a user's mailbox. This words are used with constructed social relation from the search engines world wide web for profiling and detect user intentions. Moreover, the mechanisms have proved its ability to distinguish between spam and non-spam emails [19]. Hao et al. [20] proposed an adaptive system to detect email scams (phishing emails) which deceive users to access a detrimental website. These emails convince users with friendly messages and fake personality in multiple ways. The adaptive context modeling method is consists of Prediction by Partial Matching (PPM) and suffix trees method which represent a high accuracy in deception detection. It shows good results in distinguishing between trusty and scam emails by the email data set obtained for the evaluation. Harini and Uppe Nanaji [21] developed a new system to overcome spam problem. Personalized E-mail Priorities deduct user preferences and priorities to determine important received email for drawing user's attention. The system depends on personal social networks analysis to model user preferences by multiple techniques (i.e. social network analysis, unsupervised clustering, semi-supervised feature induction and supervised classification). Quang et al. [22] proposed personalized framework against email spam by defining a new way for filtering emails. This technique of recommender system is applied through using statistical classification methods of the content and user actions. The multi-class classification is used instead of only two-class (ham and spam). In which, each class performs one action from the user (read, delete and reply). The framework was tested and shows promising results. 
Regarding email overload, according to Cox et al. [23], some existing systems such as Gmail and Immersion, that was developed by MIT Media Labs, offer tools which enable the user to determine his unique behavioral patterns. It presents certain metrics (top sender, top recipient, word count, daily inbox volume... etc.) to produce a graphical file of social network analysis about one email communications and its historical changes through consecutive times. The timely report supports the user to compare the extracted data and manage the messages efficiently upon them. According to Cecchinato, using personalized and customized tools has proved the system efficiency in dealing with email overload. Email Game is one example of personalized email tools. It encourages the user to reply to each message in his mailbox by assigning a default time. Whenever the user can accomplish all messages in the mailbox within exactly three minutes, he will be reward by giving him more scores and a smiley face as an expression of his grade. In addition to personalized tools, there are some customizable tools, which have the advantage to support the user in managing and dealing with his mailbox. There is a many tools such as Boomerang apps that enable the user to schedule messages to be sent and received or to differ/deliver the inbox message at a specific date in the future. Some websites such as Calmbox.me have customizable tools that reply to emails twice a day. It gives the user some advice and recommendations on how to best deal with his messages and how to customize email signature or change email behavior [11]. Magdalena et al. proposed MIND a pro-active documents model to solve information overload problems for organizational workers. Considering the importance of document exchange via email messages and interactive between users devices, MIND has a potential to improve email system based on integration patterns. The combined patterns including, local system services, embedded document functionality, and external services are integrated to represent a smart email system and facilitate documents exchanging [24].

\section{RESEARCH METHODOLOGY}

This investigation study focuses on university students and faculty members from their perspective about university email usage. It is concerning the general email usage for both learning and university obligation activity. Therefore, the study is dedicated to explore students and faculty members attitude toward how important the use of university email system. However, interestingly, there is a difference in perspective between the sample groups. We believe that it considered as a clue to understanding the gaps between their academic communications needs.

\subsection{Sample and study instrument}

The study population consists of students and faculty members at King Abdulaziz University. Two surveys were conducted (faculty members and students) to collect the data. The survey was conducted in November and December 2016 form 1948 respondents (i.e. 1847 students (1359 female and 488 male), and 101 faculty members (58 female, and 43 male)).

\subsection{Sampling technique}

The study used a convenience approach to cover both drown samples. The predefined criteria were constructed to suit students and faculty members representations from different majors and academic ranks. Both samples were willing and accessible to participate, and they informed that their participation is totally voluntary. The electronic survey was published through King Abdulaziz University Blackboard system to both students and faculty members.

\subsection{Surveys structures}

Table 1 shows the survey structure of students and faculty members' commitment to using university email. Both surveys were divided into five sections and consists of 17 questions. The first section consists of three questions that represent the personal information. The second section of the questionnaire consists of one question that considered the surveys main question and specifies any sections available for the user depending on his answer. The third section consists of three question that concerns the extent of the use of university email. The fourth section concerns the user perspective about the university email and some potential personalized techniques. The final section contains an open question about any suggestions or recommendations.

\section{RESULTS AND DISCUSSIONS}

By starting to analyze the students' demographic/personal data, the results show that females represent $73.6 \%$ of respondents as compared to the males who represent $26.4 \%$ of respondents. Students ages range between 19 to 25 representing $67.9 \%$ of respondents. In contrast, by analyzing the faculty members' demographic/personal data; the results show that females represent $58 \%$ of respondents as compared to $42 \%$ of male respondents. The major faculty members ages range between 35 to 40 and represent $30 \%$, the ages range between 30 to 35 ; and 25 to 30 represent $22 \%$ and $18 \%$ respectively.

Table 1 shows the distribution of respondents by faculties for both students and faculty members survey. Of all the students respondents, 39.4\% from the Foundation Year. There is a correlation among the three colleges of Economics and Management (FEM), Arts and Human Science (FAHS), and Computing and Information Technology (FCIT) in which they share the higher percentage of respondents in both questionnaires. Specifically, it was found from the students' data that $31.2 \%$ of respondents were from FEM students, followed by $13.5 \%$ of respondents from FAHS students, then $8.3 \%$ of respondents were from FCIT students, and $7.7 \%$ from other colleges. In contrast, faculty members' data is as the following: $27.6 \%$ of from FEM, then $18.4 \%$ of respondents are from FAHS, $10.2 \%$ of respondents are from FCIT, followed by a symmetrical percentage of $5.1 \%$ respondents from both Deanship of Graduate Studies (DGS) and ELearning (DEL) respondents. Finally, 33.7\% faculty members respondents are distributed in several colleges.

Table 1. Faculty usage toward University Email

\begin{tabular}{|c|c|c|}
\hline Faculties & Students & Faculty \\
\hline FCIT & $8.2 \%$ & $13.60 \%$ \\
\hline FAHS & $8.4 \%$ & $25.90 \%$ \\
\hline FEM & $25.2 \%$ & $23.50 \%$ \\
\hline DGS & $0.0 \%$ & $6.20 \%$ \\
\hline DEL & $0.0 \%$ & $6.20 \%$ \\
\hline Foundation Year & $43.1 \%$ & $0.00 \%$ \\
\hline Others & $15.2 \%$ & $24.70 \%$ \\
\hline
\end{tabular}

Figure 1 shows the results of students and faculty members utilization of university email. The results indicate that $39.5 \%$ 
of students never and rarely use the university email, $37.3 \%$ of students sometimes use it, and only $23.2 \%$ of the students always use the university email. In contrast to faculty members who represent $80.2 \%$ of the respondents that always use the university email, $8.9 \%$ sometimes use it, and $10.9 \%$ never and rarely using the university email.

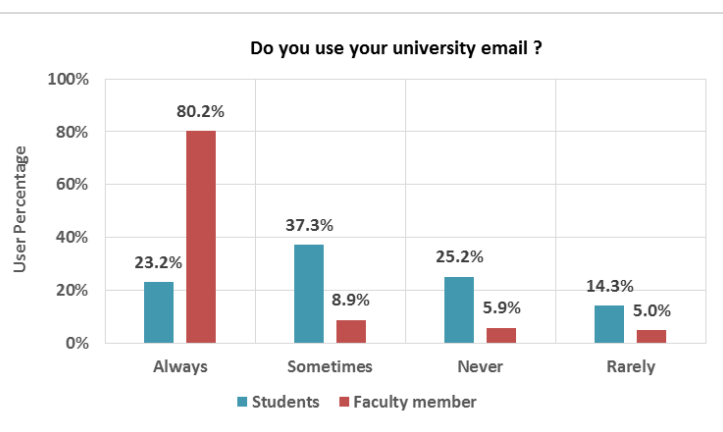

Fig. 1: University email usage.

Table 2 shows that half of the respondents students are from the Foundation Year students and represent $43.1 \%$ of the total respondents, followed by the FEM with $25.2 \%$ respondents, then the FAHS with $8.4 \%$ respondents, finally, the FCIT represents $8.2 \%$ of respondents who always use the university email. On the other hand, $25.9 \%$ of respondents from faculty members, who always use the university email, are from the FAHS, followed by the FEM with $23.5 \%$ of respondents, lastly with $13.6 \%$ from the FCIT.

Table 2. Questionnaire respondents according to faculty

\begin{tabular}{|c|c|c|}
\hline Faculties & Students & Faculties \\
\hline FCIT & $8.3 \%$ & $11.9 \%$ \\
\hline FAHS & $13.5 \%$ & $23.8 \%$ \\
\hline FEM & $31.2 \%$ & $28.7 \%$ \\
\hline DGS & $0.0 \%$ & $5 \%$ \\
\hline DEL & $0.0 \%$ & $5 \%$ \\
\hline $\begin{array}{c}\text { Foundation } \\
\text { Year }\end{array}$ & $39.4 \%$ & $0.0 \%$ \\
\hline Others & $7.7 \%$ & $25.7 \%$ \\
\hline
\end{tabular}

Figure 2 shows that $27.8 \%$ of students always use the university email in communicating and sending content to college and faculty. Besides, $74.2 \%$ of students never use it for any communication with friends and family. Moreover, $31.4 \%$ of students strongly agree that using the university email is effective as regular emails such as yahoo and google. On the other hand, $55.7 \%$ of faculty members always use the university email in communicating and sending content to college and university. About $48.9 \%$ of faculty members rarely use it for any communication with friends and family. Furthermore, $52.3 \%$ of faculty members strongly agree that using university email is effective as regular emails such as Yahoo and Google.

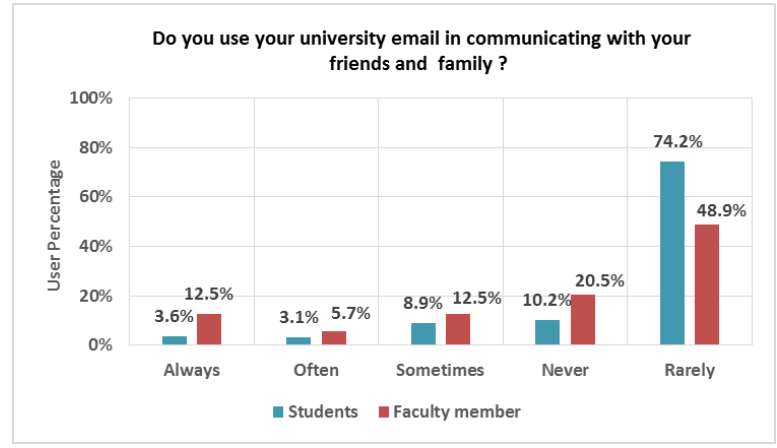

Fig. 2: University email usage to communicate with friends and family

Figure 3 shows a negative question presented to students and faculty members to confirm users' perspective about the university email usage. The results indicated that more than half of the Faculty members (61.4\%) are strongly disagree with neglecting university email as a one of the main communication channel with students, whereas only $32.8 \%$ of students are strongly disagree.

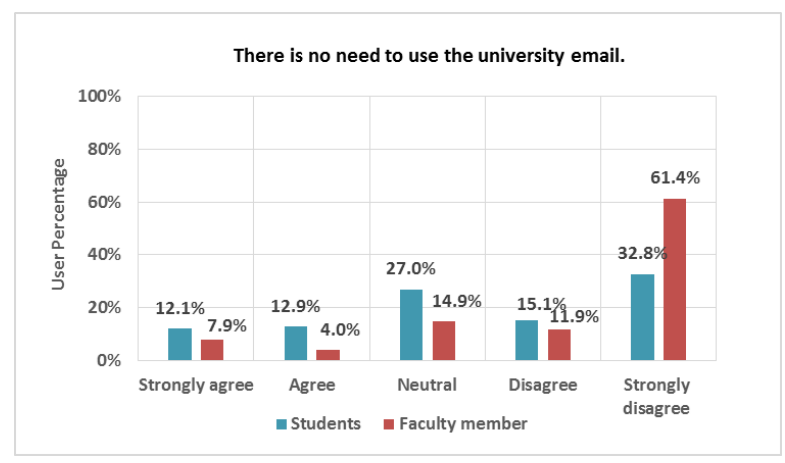

Fig. 3: Faculty members and students perspective about the university email usage

For the fourth questions, figure 4 shows that the majority of both faculty members $(51.5 \%)$ and students (50.3\%) strongly agree that receiving the news and announcements about the university in general and the college in particular through email messages will contribute to increasing the use of university email.

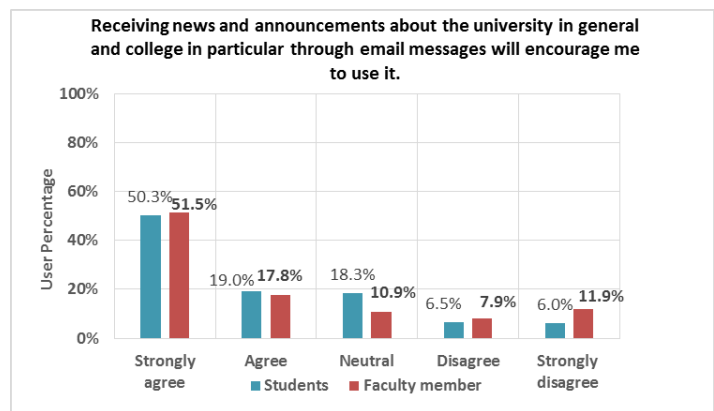

Fig. 4. Receiving the news and announcements

Figure 5 shows that both faculty members $(68.3 \%)$ and students $(60.2 \%)$ strongly agree that classifying all incoming emails by (university /college/department/job title/Section number in current semester) on the university email will contribute to increasing email usage. 


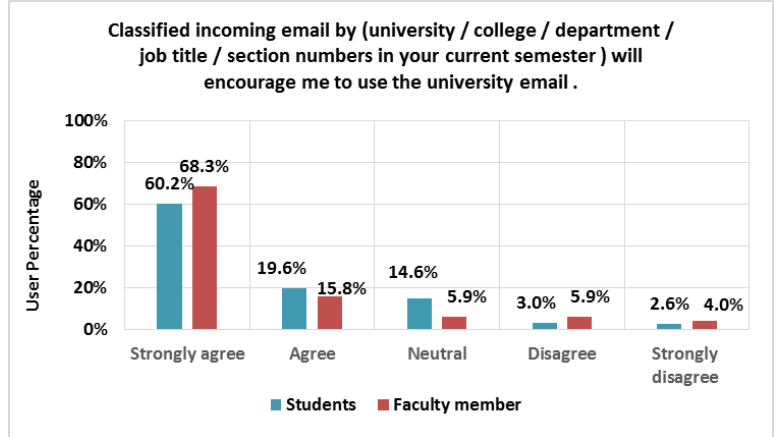

Fig. 5. Classified all incoming emails

Figure 6 shows that the majority of responses on the fourth question are also strongly agree from both students (37.2\%) and faculty members (55.4\%). They strongly agree on the possibility of adding users own signature for all sent emails as an image containing (name/college/section/personal information) on the university email will contribute to increase the email usage.

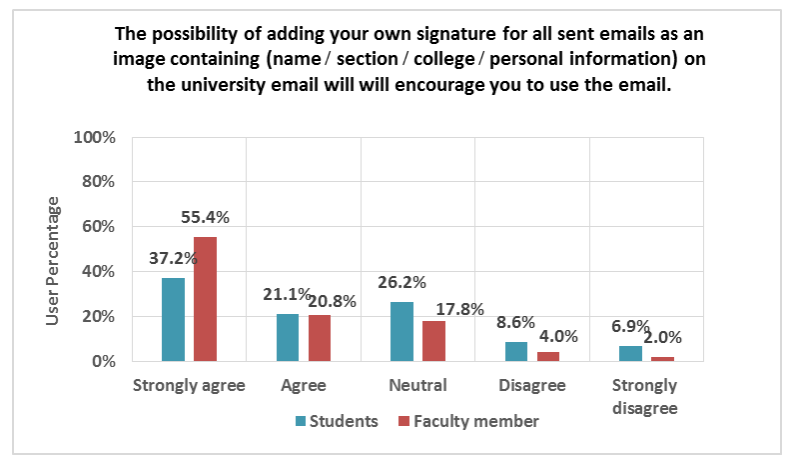

Fig. 6: Adding special signature to the university email

Figure 7 shows that the majority responses on the fifth question (57.6\% of students and $66.3 \%$ of faculty members) declare that sending all announcements and news about users section and college periodically at the university email will contribute to increasing the email usage have performed the higher percentage.

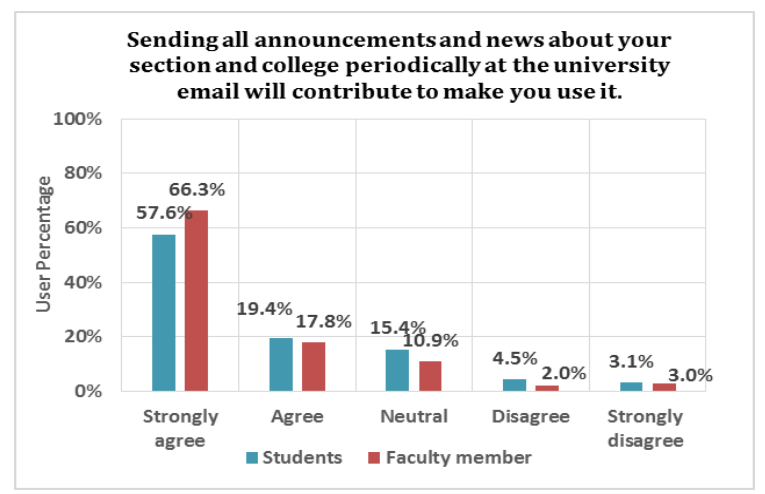

Fig. 7: Receiving news and announcements.

Figure 8 shows that the majority of both students (54.4\%) and faculty members $(62.4 \%)$ are strongly agree that having customization feature to control all news and announcements that are received about the (university /college/ section) will contribute to increase the email usage.

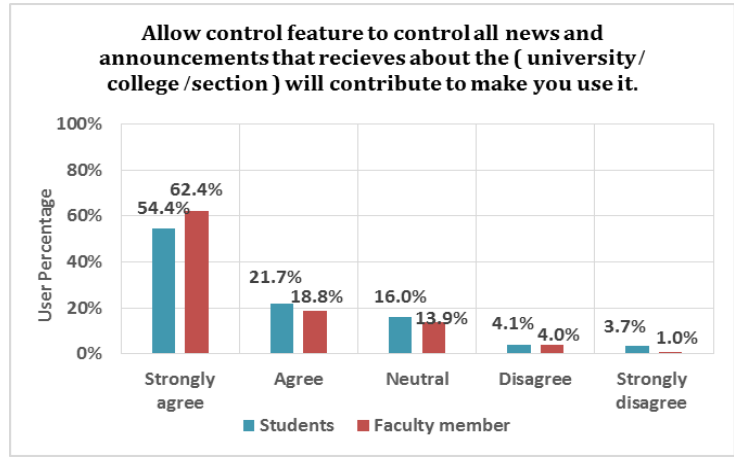

Fig. 8: Existing control feature to control all news and announcements in the University email

Figure 9 shows that $45.2 \%$ of students and $56.4 \%$ of faculty members strongly agree on the possibility that arranging all incoming emails automatically by the system to different sections as (university/college/department/general emails) will increase email usage.

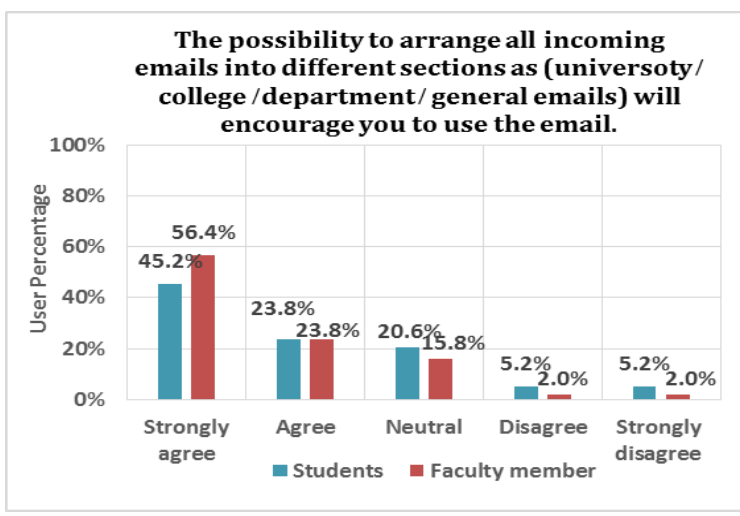

Fig. 9: Customize incoming emails.

Figure 10 shows that $41.8 \%$ of students and $55.4 \%$ of faculty members strongly agree on Synchronizing the university email with all other users email accounts such as yahoo and getting all the messages to university email box will increase the email usage.

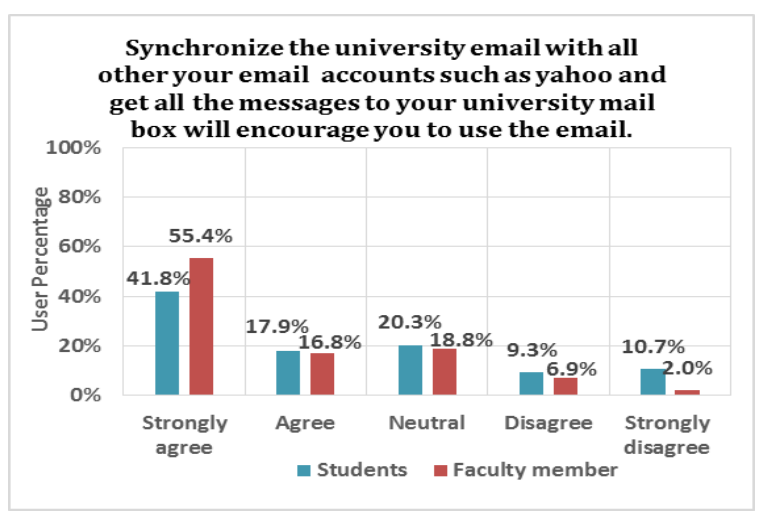

Fig. 10: Synchronize the university email with other email accounts

Figure 11 shows that responses on the last question are majorly strongly agree in both students $(40.1 \%)$ and faculty members $(51.5 \%)$. They strongly agree on the possibility of changing the interface system to attractive themes will contribute to increase the university email usage. 


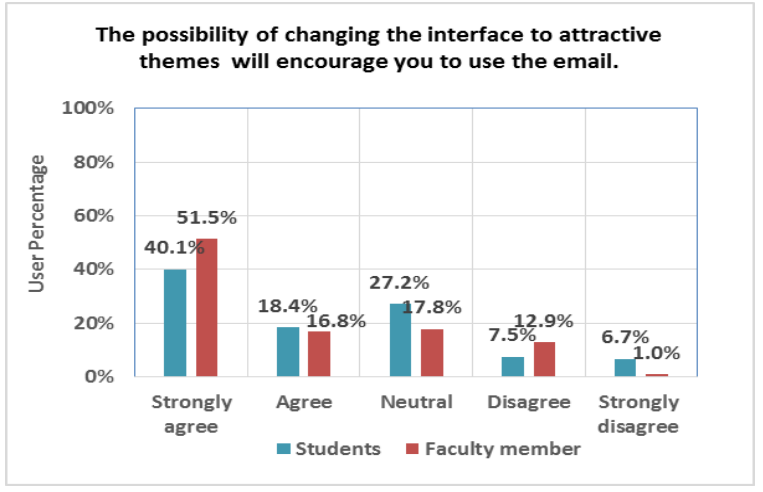

Fig. 11: Changing the email interface to attractive themes

Some faculty members and students suggestions:

- "Apply small icon within Blackboard or Odus system called (my email) will help them to use it efficiently."

- "Place updated list of emails of all university deanships, technical support for Blackboard and professors to allow students to communicate with them quickly."

- "Linking the university calendar with university email and send alerts as email messages and directly appear all the necessary appointments for us by the timeframe such as lectures duties, important dates, payment fees, the deletion and addition of our learning materials, and receive email messages about lectures, homework, and exams."

- "Setting (times, date, days) of (lectures, tests, official holidays, the start and end of the study) on email agenda automatically will be very helpful."

\section{CONCLUSION AND FUTURE WORK}

The present study was conducted to investigate students and faculty member commitment of using university email system and to explore the effects of personalized technique on increasing the email usage. Two surveys of 1948 respondents were conducted for students and faculty members. The respondents were 1847 students and 101 faculties participate from King Abdulaziz University. The most obvious finding from this study revealed that there is a difference between students and faculty members' commitment of using the university email; the results show that only $23.2 \%$ of the students always use the university email, unlike the faculty members who shows $80 \%$ commitment. Furthermore, the evidence from this study suggests that applying personalized techniques has a strong and positive impact on both types of respondents. Thus, it can be considered as a clue for a potential system improvement to increase users commitment of using the university email. The contribution of this study rises from the positive findings that can be highlighted as guidelines to support the decision makers of the higher educational institutions in designing the suitable email system. As a future work, we intend to apply the personalized technique in the university email and measure students and faculty members' attitude toward the improved system, and specify which of the personalization approach (Adaptive or Adaptable) have more positive effect than the other one in rising student commitment of using university email in King AbdulAziz University. Another possible area of future research would be to investigate whether combining the two personalized approaches (adaptive and adaptable) in a mixedinitiative design may improve the usage of university email system and raise its efficiency and increase better communication through it

\section{REFERENCES}

[1] L. Li and J. P. Pitts, "Does it really matter? Using virtual office hours to enhance student-faculty interaction," Journal of Information Systems Education, vol. 20, p. $175,2009$.

[2] K. Betts, "Lost in translation: Importance of effective communication in online education," Online Journal of Distance Learning Administration, vol. 12, pp. 1-13, 2009.

[3] H. Alkahtani, R. Dawson, and R. Lock, "Communication and effective email usage in Saudi Arabia," 2015.

[4] J. P. Mendez, J. Curry, M. Mwavita, K. Kennedy, K. Weinland, and K. Bainbridge, "To friend or not to friend: Academic interaction on Facebook," International Journal of Instructional Technology \& Distance Learning, vol. 6, pp. 33-47, 2009.

[5] L. Robson, L. Cook, and N. Habgood, "Student experience of university email communication," in Proceedings of the 10th International Technology, Education and Development Conference, 2016, pp. 6118-6124.

[6] J. Waycott, S. Bennett, G. Kennedy, B. Dalgarno, and K. Gray, "Digital divides? Student and staff perceptions of information and communication technologies," Computers \& education, vol. 54, pp. 1202-1211, 2010.

[7] T. Judd and G. Kennedy, "A five-year study of oncampus Internet use by undergraduate biomedical students," Computers \& Education, vol. 55, pp. 15641571,2010

[8] C. Rasmussen and G. Johnson, "The Ripple Effect of Virginia Tech: Assessing the Nationwide Impact on Campus Safety and Security Policy and Practice," Midwestern Higher Education Compact, 2008.

[9] M. D. Roblyer, M. McDaniel, M. Webb, J. Herman, and J. V. Witty, "Findings on Facebook in higher education: A comparison of college faculty and student uses and perceptions of social networking sites," The Internet and higher education, vol. 13, pp. 134-140, 2010.

[10] C. McLoughlin and M. J. Lee, "Future learning landscapes: Transforming pedagogy through social software," Innovate: Journal of Online Education, vol. 4, p. 1, 2008.

[11] M. E. Cecchinato, J. Bird, and A. L. Cox, "Personalised email tools: a solution to email overload?," in CHI'14 Workshop: Personalised Behaviour Change Technologies, 2014.

[12] E. Carmel and E. Káganer, "Ayudarum: an Austrian crowdsourcing company in the Startup Chile accelerator program," Journal of Business Economics, vol. 84, pp. 469-478, 2014

[13] L. Singh and G. Chetty, "Email Personalization and User Profiling Using RANSAC Multi Model Response Regression Based Optimized Pruning Extreme Learning Machines and Gradient Boosting Trees," in International 
Conference on Neural Information Processing, 2015, pp. 302-309.

[14] M. Srivastava and M. Goyal, "Personalization of automatic e-mail response for the university system," in Confluence 2013: The Next Generation Information Technology Summit (4th International Conference), 2013, pp. 485-489.

[15] K. Karthik and R. Ponnusamy, "Adaptive machine learning approach for emotional email classification," in International Conference on Human-Computer Interaction, 2011, pp. 552-558.

[16] H. Sethi, A. Sirohi, and M. K. Thakur, "Intelligent Mail Box," in Information Systems Design and Intelligent Applications, ed: Springer, 2016, pp. 441-450.

[17] B. Serim, V. T. Tung, T. Ruotsalo, L. Micallef, and G. Jacucci, "mailVis: Visualizing emailbox for re-finding emails," in Visual Analytics Science and Technology (VAST), 2014 IEEE Conference on, 2014, pp. 275-276.

[18] J. BRZEZINSKI and M. DAIN, "MEME': An Adaptive Email-based Knowledge," in Advanced Computer Systems: Eighth International Conference, ACS'2001 Mielno, Poland October 17-19, 2001 Proceedings, 2012, p. 171.

[19] C.-H. Mao, H.-M. Lee, and C.-F. Yeh, "Adaptive e-mails intention finding system based on words social networks," Journal of Network and Computer Applications, vol. 34, pp. 1615-1622, 2011.

[20] P. Hao, X. Chen, N. Cheng, R. Chandramouli, and K. Subbalakshmi, "Adaptive context modeling for deception detection in emails," in International Workshop on Machine Learning and Data Mining in Pattern Recognition, 2011, pp. 458-468.

[21] K. Harini and U. Nanaji, "Implementation of personalized email prioritization-A content based social network analysis," International Journal of Computer Science and Communication Networks, vol. 1, pp. 218 $221,2012$.

[22] Q. M. Ha, Q. A. Tran, and T. T. Luyen, "Personalized email recommender system based on user actions," in Asia-Pacific Conference on Simulated Evolution and Learning, 2012, pp. 280-289.

[23] A. L. Cox, J. Bird, and R. Fleck, "Digital Epiphanies: how self-knowledge can change habits and our attitudes towards them," 2013.

[24] M. Godlewska and B. Wiszniewski, "Smart Email: Almost An Agent Platform," in Innovations and Advances in Computing, Informatics, Systems Sciences, Networking and Engineering, ed: Springer, 2015, pp. 581-589. 